\title{
The quark distributions of baryons
}

\author{
Bo-Qiang $\mathrm{Ma}^{\mathrm{a} *}$
}

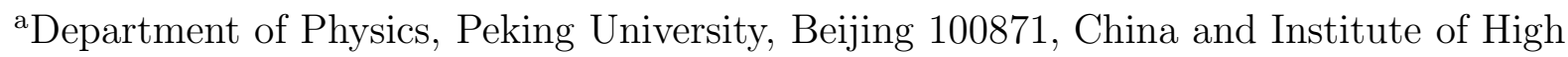
Energy Physics, Academia Sinica, P. O. Box 918(4), Beijing 100039, China

The recent progress in the investigations on the quark structure of the $\Lambda$ and $\Sigma$ baryons are reviewed. It is shown that the quark structure of $\Lambda$ and $\Sigma$ hyperons can provide a new domain to test various theories concerning the spin and flavor structure of the nucleon. The $\Lambda$ and $\Sigma$ Physics might be new directions to explore the quark distributions of baryons both theoretically and experimentally.

\section{Introduction}

The nucleons were considered as point-like particles when they were first isolated in the 30 's and later on they were found to have extended structure in the 50's. The quark model suggested the nucleons as composite systems of more basic constituents of quarks and the lepton nucleon deep elastic scattering experiments in the latter 60's confirmed the quarkparton structure of nucleons and led to a new era to consider the nucleons in terms of quark and gluon degrees of freedom in QCD. After more than three decades experimental and theoretical investigations of various deep inelastic scattering processes, the structure of the nucleons has been found to be more complicated than naively expected and there have been many surprises found in recent years concerning the sea quark content of the nucleons:

- There have been an extensive attention on the spin content of the proton raised by the observation of the Ellis-Jaffe sum rule violation and a much smaller helicity sum of quarks than naively expected [1,2].

- The observation of the Gottfried sum violation suggested a flavor asymmetry of $u$ and $d$ sea quarks inside the proton [3], or alternative possibility of including also some isospin symmetry breaking between proton and neutron [1].

- The current knowledge of the strange quark content of the proton is still very poor, since one is still unclear as to whether or not strange quarks are highly polarized inside the proton [5,6], and it is even more obscure whether or not the strange quark-antiquark distributions are symmetric [6].

It was commonly taken for granted that we understand better of the valence quark structure of the nucleons. However, the recent progress shows that the flavor and spin 
structure of the valence quarks is still not clear at $x=1$. For example, there are different predictions concerning the ratio $d(x) / u(x)$ at $x \rightarrow 1$ from the perturbative QCD (pQCD) analysis [7,8] and the SU(6) quark-diquark model [9 [1], and there are different predictions concerning the value of $F_{2}^{n}(x) / F_{2}^{p}(x)$ at large $x$, which has been taken to be $1 / 4$ as in the quark-diquark model in most parameterizations of quark distributions. Recent analysis [11, [12] of experimental data from several processes suggests that $F_{2}^{n}(x) / F_{2}^{p}(x) \rightarrow 3 / 7$ as $x \rightarrow 1$, in favor of the pQCD prediction. The spin structure of the valence quarks is also found to be different near $x=1$ in these models, and predictions have been made concerning the non-dominant valence down $(d)$ quark, so that $\Delta d(x) / d(x)=-1 / 3$ in the quark-diquark model [10,11], a result which is different from the pQCD prediction $\Delta q(x) / q(x)=1$ for either $u$ and $d\|8\|$. At the moment, there is still no clear data in order to check these different predictions, although the available measurements [13,14 for the polarized $d$ quark distributions seem to be negative at large $x$, slightly in favor of the quark-diquark model prediction.

It is important to perform high precision measurements of available physical quantities and/or to measure new quantities related to the flavor and spin structure of the nucleons, in order to have a better understanding of the quark-gluon structure of the nucleon. However, it should be more meaningful and efficient if we can find a new domain where the same physics concerning the structure of the nucleons can manifest itself in a way that is more easy and clean to be detected and checked. It has been recently suggested by Schmidt, Yang, and I [15, 16] that the quark structure of the $\Lambda$ and the $\Sigma$ are such new frontiers that can be used to test various ideas concerning the structure of the nucleons. In this talk I will review some most recent progress in this direction and review a case study [16] to check the theory on the quark distributions of the nucleons from the quark distributions of the $\Lambda$ 's.

\section{The quark structure of the $\Lambda$}

There have been many theoretically studies concerning the quark structure of the $\Lambda$. It was found by Burkardt and Jaffe [17] that the $u$ and $d$ quarks inside a $\Lambda$ should be negatively polarized from SU(3) symmetry. It was also pointed out by Soffer and I [18] that the flavor and spin content of the $\Lambda$ can be used to test different predictions concerning the spin structure of the nucleon and the quark-antiquark asymmetry of the nucleon sea. Most recently, Schmidt, Yang and I found [15, 16] that the flavor and spin structure of the $\Lambda$ near $x=1$ can provide clean tests between perturbative QCD (pQCD) and the $\mathrm{SU}(6)$ quark-diquark model predictions. We also found that the non-dominant up $(u)$ and down $(d)$ quarks should be positively polarized at large $x$, even though their net spin contributions to the $\Lambda$ might be zero or negative. There have been calculations of the explicit shapes for the quark fragmentation functions in a quark-diquark model [19] and for the quark distributions inside the $\Lambda$ in the MIT bag model [20]. The sea quark content of the $\Lambda$ has been also studied by Boros, Londergan and Thomas and novel features in similar to the nucleon case have been suggested [20]. Thus it is clear that the quark structure of $\Lambda$ is a frontier which can enrich our understanding concerning the flavor and spin structure of the nucleons and provides a new domain to test various ideas concerning the hadron structure that come from the available nucleon studies. 
Direct measurement of the quark distributions of the $\Lambda$ is difficulty, since the $\Lambda$ is a charge-neutral particle which cannot be accelerated as incident beam and its short life time makes it also difficult to be used as a target. However, the quark distributions and the quark fragmentation functions are interrelated quantities that can uncover the structure of the involved hadron. For example, we know that the quark distributions inside a hadron are related by crossing symmetry to the fragmentation functions of the same flavor quark to the same hadron, by a simple reciprocity relation [21]

$$
q_{h}(x) \propto D_{q}^{h}(z)
$$

where $z=2 p \cdot q / Q^{2}$ is the momentum fraction of the produced hadron from the quark jet in the fragmentation process, and $x=Q^{2} / 2 p \cdot q$ is the Bjorken scaling variable corresponding to the momentum fraction of the quark from the hadron in the DIS process. Although such an approximate relation may be only valid at a specific scale $Q^{2}$ near $x=1$ and $z=1$ at leading order approximation, it can provide a reasonable connection between different physical quantities and lead to different predictions about the fragmentations based on our understanding of the quark structure of a hadron [18,22]. Thus we can use various $\Lambda$ fragmentation processes to test different predictions.

In principle we can test the different predictions by a measurement of a complete set of quark to $\Lambda$ fragmentation functions. However, in practice we do not need such systematic studies of quark to $\Lambda$ fragmentations before we can test the different predictions. Many processes have been suggested to measure various quark to $\Lambda$ fragmentation functions:

- Jaffe and Burkardt [17 suggested one promising method to obtain a complete set of polarized fragmentation functions for different quark flavors based on the measurement of the helicity asymmetry for semi-inclusive production of $\Lambda$ hyperons in $e^{+} e^{-}$annihilation on the $Z^{0}$ resonance.

- Measurements of the light-flavor quark fragmentations into $\Lambda$ have been also suggested from polarized electron DIS process [23] and neutrino DIS process [24], based on the $u$-quark dominance assumption.

- There is also a recent interesting suggestion to determine the polarized fragmentation functions by measuring the helicity transfer asymmetry in the process $p \vec{p} \rightarrow$ $\vec{\Lambda} X[25$.

- More recently, Soffer and I suggested [18] to measure a complete set of quark to $\Lambda$ unpolarized and polarized fragmentation functions for different quark flavors by the systematic exploitation of unpolarized and polarized $\Lambda$ and $\bar{\Lambda}$ productions in neutrino, antineutrino and polarized electron DIS processes.

Thus we have a new and rich domain from where we can study the quark structure of the $\Lambda$ both theoretically and experimentally. For example, a recent detailed analysis [16] of the available $\Lambda$-polarization data in $e^{+} e^{-}$annihilation at the $Z$-pole supports the prediction [15] that the $u$ and $d$ quarks inside the $\Lambda$ should be positively polarized at large $x$, though their net helicities might be zero or negative. 


\section{The quark structure of the $\Sigma$}

Although the $\Lambda$ can provide a clean test of the different flavor and spin structure between different models, we still need a connection between the quark distributions inside the $\Lambda$ and the quark fragmentation into a $\Lambda$ and such a connection is not completely free from theoretical and experimental uncertainties. Thus it is meaningful to find a charged baryon which has also different flavor and spin structure between different models. The charged baryons other than nucleons, such as $\Sigma^{ \pm}$, may be used as beam to directly measure their own quark structure in case the structure of the target is comparatively well known. Using the $\Sigma^{ \pm}$as beam in Drell-Yan processes has been suggested [26] for the purpose of studying the flavor asymmetry in the sea of the baryons, and the sea quarks of the $\Sigma$ 's have been discussed [20,26]. It has been recently found by Schmidt, Yang and I [27] that the $\Sigma$ 's have the most significant difference in the flavor and spin structure of the valence quarks between the quark-diquark model and pQCD at medium to large $x$, and the measurement of Drell-Yan process for $\Sigma^{ \pm}$beams on the isoscalar targets can test different predictions of the quark structure of the $\Sigma^{ \pm}$baryons. It is also pointed out by Cao and Signal [28] recently that there are also quark-antiquark asymmetries in the $\Sigma$ 's, i.e, for $d-\bar{d}$ distributions inside $\Sigma^{+}$(uus) and for $u-\bar{u}$ distributions inside $\Sigma^{-}(d d s)$, in analogy to the strangeness quark-antiquark distribution asymmetry inside the nucleon from the baryonmeson fluctuation model [6]. It should be interesting if one can find a physical quantity that can measure and test such quark-antiquark distribution asymmetries inside the $\Sigma$ 's. Thus the quark structure of the $\Sigma$ 's can be also a new domain to test different theories concerning the quark distributions of the nucleons.

\section{A case study}

As a case study to show that the measurement of the quark structure of the $\Lambda$ may serve to test theory concerning the quark structure of the nucleon, we present a brief review on the spin and flavor structure of the valence quarks for the nucleon and the $\Lambda$ in a light-cone $\mathrm{SU}(6)$ quark-spectator-diquark model [10,15,16].

As we know, it is proper to describe deep inelastic scattering as the sum of incoherent scatterings of the incident lepton on the partons in the infinite momentum frame or in the light-cone formalism. The unpolarized valence quark distributions $u_{v}(x)$ and $d_{v}(x)$ of the nucleon are given in this model [10] by

$$
\begin{aligned}
& u_{v}(x)=\frac{1}{2} a_{S}(x)+\frac{1}{6} a_{V}(x) ; \\
& d_{v}(x)=\frac{1}{3} a_{V}(x),
\end{aligned}
$$

where $a_{D}(x)$ ( $D=S$ for scalar spectator or $V$ for axial vector spectator) is normalized such that $\int_{0}^{1} \mathrm{~d} x a_{D}(x)=3$, and it denotes the amplitude for quark $q$ to be scattered while the spectator is in the diquark state $D$. Exact $\mathrm{SU}(6)$ symmetry provides the relation $a_{S}(x)=a_{V}(x)$, which implies the valence flavor symmetry $u_{v}(x)=2 d_{v}(x)$. This gives the prediction $F_{2}^{n}(x) / F_{2}^{p}(x) \geq 2 / 3$ for all $x$, which is ruled out by the experimental observation $F_{2}^{n}(x) / F_{2}^{p}(x)<1 / 2$ for $x \rightarrow 1$. The SU(6) quark-diquark model [9] introduces a breaking to the exact $\mathrm{SU}(6)$ symmetry by the mass difference between the scalar and 
vector diquarks and predicts $d(x) / u(x) \rightarrow 0$ at $x \rightarrow 1$, leading to a ratio $F_{2}^{n}(x) / F_{2}^{p}(x) \rightarrow$ $1 / 4$, which could fit the data and has been accepted in most parameterizations of quark distributions for the nucleon. It has been shown that the SU(6) quark-spectator-diquark model can reproduce the $u$ and $d$ valence quark asymmetry that accounts for the observed ratio $F_{2}^{n}(x) / F_{2}^{p}(x)$ at large $x$ [10]. This supports the quark-spectator picture of deep inelastic scattering in which the difference between the mass of the scalar and vector spectators is essential in order to reproduce the explicit $\mathrm{SU}(6)$ symmetry breaking while the bulk SU(6) symmetry of the quark model still holds.

The quark helicity distributions for the $u$ and $d$ quarks can be written as [10]

$$
\begin{aligned}
& \Delta u_{v}(x)=u_{v}^{\uparrow}(x)-u_{v}^{\downarrow}(x)=-\frac{1}{18} a_{V}(x) W_{q}^{V}(x)+\frac{1}{2} a_{S}(x) W_{q}^{S}(x) ; \\
& \Delta d_{v}(x)=d_{v}^{\uparrow}(x)-d_{v}^{\downarrow}(x)=-\frac{1}{9} a_{V}(x) W_{q}^{V}(x),
\end{aligned}
$$

in which $W_{q}^{S}(x)$ and $W_{q}^{V}(x)$ are the Melosh-Wigner correction factors 29 for the scalar and axial vector spectator-diquark cases. From Eq. (2) one gets

$$
\begin{aligned}
& a_{S}(x)=2 u_{v}(x)-d_{v}(x) \\
& a_{V}(x)=3 d_{v}(x) .
\end{aligned}
$$

Combining Eqs. (3) and (4) we have

$$
\begin{aligned}
& \Delta u_{v}(x)=\left[u_{v}(x)-\frac{1}{2} d_{v}(x)\right] W_{q}^{S}(x)-\frac{1}{6} d_{v}(x) W_{q}^{V}(x) \\
& \Delta d_{v}(x)=-\frac{1}{3} d_{v}(x) W_{q}^{V}(x) .
\end{aligned}
$$

Thus we arrive at simple relations [10] between the polarized and unpolarized quark distributions for the valence $u$ and $d$ quarks. The calculated polarization asymmetries $A_{1}^{N}=2 x g_{1}^{N}(x) / F_{2}^{N}(x)$, including the Melosh-Wigner rotation, have been found 10 to be in reasonable agreement with the experimental data, at least for $x \geq 0.1$. A large asymmetry between $W_{q}^{S}(x)$ and $W_{q}^{V}(x)$ leads to a better fit to the data than that obtained from a small asymmetry.

The key point that the light-cone SU(6) quark-diquark model can give a good description of the experimental observation related to the proton spin quantities relies on the fact that the quark helicity measured in polarized deep inelastic scattering is different from the quark spin in the rest frame of the nucleon or in the quark model [2,29]. Thus the observed small value of the quark helicity sum for all quarks is not necessarily in contradiction with the quark model in which the proton spin is provide by the valence quarks. From this sense, there is no serious "spin puzzle" or "spin crisis" as it was first understood. Of course, the sea quark content of the nucleon is complicated and it seems that the baryon-meson fluctuation configuration [6] composes one important part of the non-perturbative aspects of the nucleon. We should not expect that the valence quarks provide $100 \%$ of the proton spin, and the sea quarks and gluons should also contribute some part of the proton spin, thus it is meaningful to design new experimental methods to measure these contributions independently. Useful relations that can be used to measure the quark spin as meant in the quark model and the quark orbital angular momentum from a relativistic viewpoint have been discussed in Refs. [2] and [30. It has been pointed 
out [2] that the quark spin distributions $\Delta q_{Q M}(x)$ are connected with the quark helicity distributions $\Delta q(x)$ and the quark transversity distributions $\delta q(x)$ by an approximate relation:

$$
\Delta q_{Q M}(x)+\Delta q(x)=2 \delta q(x) .
$$

The quark orbital angular momentum $L_{q}(x)$ and the quark helicity distribution $\Delta q(x)$ are also found [30] to be connected to the quark model spin distribution $\Delta q_{Q M}(x)$ by a relation:

$$
\frac{1}{2} \Delta q(x)+L_{q}(x)=\frac{1}{2} \Delta q_{Q M}(x),
$$

which means that one can decompose the quark model spin contribution $\Delta q_{Q M}(x)$ by a quark helicity term $\Delta q(x)$ plus an orbital angular momentum term $L_{q}(x)$. There is also a new relation connecting the quark orbital angular momentum with the measurable quark helicity distribution and transversity distribution:

$$
\Delta q(x)+L_{q}(x)=\delta q(x),
$$

from which we may have new sum rules connecting the quark orbital angular momentum with the nucleon axial and tensor charges. The quark tranversity and orbital angular momentum distributions have been also calculated in the light-cone SU(6) quark-diquark model [2,30]. Thus future measurements of new physical quantities related to the proton spin structure can be used to test whether the framework is correct or not, and detailed predictions and discussions can be found in Refs. [2,30,31]. We point out that one of the predictions of the framework is the small helicity contribution from the anti-quarks and the available experimental data [13,14] are consistent with this prediction. This is different from most other works in which a large negative spin contribution from anti-quarks is required to reproduce the observed small quark helicity sum. In our framework the Melosh-Winger rotation [2,29] and the flavor asymmetry of the Melosh-Wigner rotation factors between the $u$ and $d$ quarks [10] are the main reason for the reduction of the quark helicity sum compared to the naive quark model prediction.

However, as I have pointed out in the Introduction, the quark distributions of the $\Lambda$ can be also used as a new domain to check the light-cone SU(6) quark-diquark model. By applying the same analysis from the nucleon case to the $\Lambda$ case by Schmidt, Yang, and I [15,16], we get the unpolarized quark distributions for the three valence $u, d$, and $s$ quarks for the $\Lambda$,

$$
\begin{aligned}
& u_{v}(x)=d_{v}(x)=\frac{1}{4} a_{V(q s)}(x)+\frac{1}{12} a_{S(q s)}(x) ; \\
& s_{v}(x)=\frac{1}{3} a_{S(u d)}(x),
\end{aligned}
$$

where $a_{D\left(q_{1} q_{2}\right)}(x) \propto \int\left[\mathrm{d}^{2} \vec{k}_{\perp}\right]\left|\varphi\left(x, \vec{k}_{\perp}\right)\right|^{2}(D=S$ or $V)$ denotes the amplitude for the quark $q$ being scattered while the spectator is in the diquark state $D$, and is normalized such that $\int_{0}^{1} a_{D\left(q_{1} q_{2}\right)}(x) \mathrm{d} x=3$. Similar to the nucleon case, the quark spin distributions for the three valence quarks can be expressed as,

$$
\begin{aligned}
& \Delta u_{v}(x)=\Delta d_{v}(x)=-\frac{1}{12} a_{V(q s)}(x) W_{V(q s)}(x)+\frac{1}{12} a_{S(q s)}(x) W_{S(q s)}(x) \\
& \Delta s_{v}(x)=\frac{1}{3} a_{S(u d)}(x) W_{S(u d)}(x),
\end{aligned}
$$


where $W_{D}(x)$ is the correction factor due to the Melosh-Wigner rotation. It has been shown 15,16] that the differences in the diquark masses $m_{S(u d)}, m_{S(q s)}$, and $m_{V(q s)}$ cause the symmetry breaking between $a_{D\left(q_{1} q_{2}\right)}(x)$ in a way that $a_{S(u d)}(x)>a_{S(q s)}(x)>a_{V(q s)}(x)$ at large $x$. Thus the quark-diquark model predicts, in the limit $x \rightarrow 1$, that $u(x) / s(x) \rightarrow 0$ for the unpolarized quark distributions, $\Delta s(x) / s(x) \rightarrow 1$ for the dominant valence $s$ quark, and also $\Delta u(x) / u(x) \rightarrow 1$ for the non-dominant valence $u$ and $d$ quarks.

Recently there have been detailed measurements of the $\Lambda$ polarizations from the $Z$ decays in $e^{+} e^{-}$-annihilation [32 34]. The measured $\Lambda$-polarization has been compared with several theoretical calculations 24, 35, 36] based on simple ansatz such as $\Delta D_{q}^{\Lambda}(z)=$ $C_{q}(z) D_{q}^{\Lambda}(z)$ with constant coefficients $C_{q}$, or Monte Carlo event generators without a clear physical motivation. Schmidt, Yang and I [16] calculated the $\Lambda$-polarization in $e^{+} e^{-}$annihilation at the $Z$-pole by connecting the quark to $\Lambda$ fragmentation functions with the quark distributions inside the $\Lambda$. It has been found [16] that the theoretical results from the quark-diquark model fit the data very well for the available $\Lambda$-polarization data in $e^{+} e^{-}$annihilation at the $Z$-pole within its present precision. Thus the quark-diquark model provides a successful description of the $\Lambda$-polarization $P_{\Lambda}(z)$, in addition to its successful descriptions of the ratio $F_{2}^{n}(x) / F_{2}^{p}(x)$ and the polarized structure functions for the proton and neutron. However, pQCD can also give a good description of the data by taking into account the suppression of quark helicities compared to the SU(6) quark model values of quark spin distributions. Thus the prediction of positive polarizations for the $u$ and $d$ quarks inside the $\Lambda$ at $x \rightarrow 1$ is supported by the available experimental data.

It is still not possible to make a clear distinction between the two different predictions of the flavor and spin structure of the $\Lambda$ by only the $\Lambda$-polarization in $e^{+} e^{-}$-annihilation near the $Z$-pole. Thus new information from other quantities related to the flavor and spin structure of the $\Lambda$ are needed before we can have a clean distinction between different predictions, and it seems that $\Lambda(\bar{\Lambda})$ production in the neutrino (anti-neutrino) DIS processes [18] are more sensitive to different flavors.

\section{Conclusion}

From the above brief review on the recent progress in the investigations on the quark structure of the $\Lambda$ and $\Sigma$ hyperons, we arrive at the conclusion that the quark structure of $\Lambda$ and $\Sigma$ hyperons can provide new domains to test various theories concerning the spin and flavor structure of the nucleon. Thus the $\Lambda$ and $\Sigma$ Physics should be new directions to explore the quark distributions of baryons both theoretically and experimentally.

This review is based on the works with my collaborators Stan Brodsky, Andreas Schäfer, Ivan Schmidt, Jacques Soffer, and Jian-Jun Yang. I would like to express my great thanks to them for the enjoyable collaborations and the encouragements from them.

\section{REFERENCES}

1. For reviews, see, e.g., H.-Y. Cheng, Int. J. Mod. Phys. A 11 (1996) 5109; G.P. Ramsey, Prog. Part. Nucl. Phys. 39 (1997) 599.

2. For a recent discussion, see, B.-Q. Ma, I. Schmidt, J. Soffer, Phys. Lett. B 441 (1998) 461. 
3. For a recent review, see, e.g., S. Kumano, Phys. Rep. 303 (1998) 183.

4. B.-Q. Ma, Phys. Lett. B 274 (1992) 111.

5. S.J. Brodsky, J. Ellis, and M. Karliner, Phys. Lett. B 206 (1988) 309; J. Ellis and M. Karliner, Phys. Lett. B 213 (1988) 73; B 341 (1995) 397.

6. See, e.g., S.J. Brodsky and B.-Q. Ma, Phys. Lett. B 381 (1996) 317, and references therein.

7. G.R. Farrar and D.R. Jackson, Phys. Rev. Lett. 35 (1975) 1416.

8. S.J. Brodsky, M. Burkardt, and I. Schmidt, Nucl. Phys. B 441 (1995) 197.

9. R. Carlitz, Phys. Lett. B 58 (1975) 345; J. Kaur, Nucl. Phys. B 128 (1977) 219; A. Schäfer, Phys. Lett. B 208 (1988) 175.

10. B.-Q. Ma, Phys. Lett. B 375 (1996) 320; B.-Q. Ma and A. Schäfer, Phys. Lett. B 378 (1996) 307.

11. W. Melnitchouk and A.W. Thomas, Phys. Lett. B 377 (1996) 11.

12. U.K. Yang and A. Bodek, Phys. Rev. Lett. 82 (1999) 2467.

13. SM Collab., B. Adeva et al., Phys. Lett. B 369 (1996) 93; B 420 (1998) 180.

14. The HERMES Collaboration, K. Ackerstaff et al., Phys. Lett. B 484 (1999) 123.

15. B.-Q. Ma, I. Schmidt, and J.-J. Yang, Phys. Lett. B 477 (2000) 107.

16. B.-Q. Ma, I. Schmidt, and J.-J. Yang, Phys. Rev. D 61 (2000) 034017.

17. M. Burkardt and R.L. Jaffe, Phys. Rev. Lett. 70 (1993) 2537.

18. B.-Q. Ma and J. Soffer, Phys. Rev. Lett. 82 (1999) 2250.

19. M. Nzar and P. Hoodbhoy, Phys. Rev. D 51 (1995) 32.

20. C. Boros and A.W. Thomas, Phys. Rev. D 60 (1999) 074017; C. Boros, J.T. Londergan, and A.W. Thomas, Phys. Rev. D 61 (2000) 014007.

21. V.N. Gribov and L.N. Lipatov, Phys. Lett. B 37 (1971) 78; Sov. J. Nucl. Phys. 15 (1972) 675.

22. S.J. Brodsky and B.-Q. Ma, Phys. Lett. B 392 (1997) 452.

23. R.L. Jaffe, Phys. Rev. D 54 (1996) R6581.

24. A. Kotzinian, A. Bravar, and D.von Harrach, Eur. Phys. J. C 2 (1998) 329.

25. D.de Florian, M. Stratmann, and W. Vogelsang, Phys. Rev. Lett. 81 (1998) 530.

26. M. Alberg et al, Phys. Lett. B 389 (1996) 367; M. Alberg, T. Falter, and E.M. Henley, Nucl. Phys. A 644 (1998) 93.

27. B.-Q. Ma, I. Schmidt, and J.-J. Yang, Nucl. Phys. B 574 (2000) 331.

28. F.-G. Cao and A.I. Signal, Phys. Lett. B 474 (2000) 138.

29. B.-Q. Ma, J. Phys. G 17 (1991) L53; B.-Q. Ma and Q.-R. Zhang, Z. Phys. C 58 (1993) 479.

30. B.-Q. Ma and I. Schmidt, Phys. Rev. D 58 (1998) 096008.

31. B.-Q. Ma and I. Schmidt, J. Phys. G: Nucl. Part. Phys. 24 (1998) L71.

32. ALEPH Collaboration, D. Buskulic et al, Phys. Lett. B 374 (1996) 319.

33. DELPHI Collaboration, Report No.DELPHI 95-86 PHYS 521, CERN-PPE-95-172, presented at the EPS-HEP 95 conference, Brussels, 1995.

34. OPAL Collaboration, K. Ackerstaff et al, Eur. Phys. J. C 2 (1998) 49.

35. C. Boros and Z. Liang, Phys. Rev. D 57 (1998) 4491.

36. D.de Florian, M. Stratmann, and W. Vogelsang, Phys. Rev. D 57 (1998) 5811. 